\title{
PSEUDO LOCALLY COMPACT SPACES ${ }^{1}$
}

\section{ANNETTE SINCLAIR}

1. Introduction. When metrics are defined in the usual ways for either the set of all functions analytic on a closed region $\bar{R}$ or the set of all functions analytic in an open region $R$, the spaces so determined have properties resembling local compactness.

For the set of functions analytic on a closed region $\bar{R}$, that is analytic at each point of $\bar{R}$, a metric $d(f, g)$ is usually defined as $\max _{z \in \bar{R}} \mid(f(z)-g(z) \mid$. Then any infinite subset of a closed bounded neighborhood $\bar{N}_{\theta}$ contains a sequence $\left\{f_{n}(z)\right\}$ which converges uniformly on any closed subset of $R[3, \text { p. } 140 ; 4, \text { p. 169 }]^{2}$ and $\lim _{n \rightarrow \infty} f_{n}(z)$ $=f(z)$ is analytic at interior points of $R$. But the convergence need not be uniform on $\bar{R}$ and $f(z)$ is not necessarily analytic on the closed region $\bar{R}$. Hence, $f$ need not belong to $\bar{N}_{q}$ and the space is not locally compact.

A metric for the set $R$ of functions analytic in an open region $R$ is sometimes defined in the following manner [3, p. 139]. Let $\left\{R_{i}\right\}$ be a monotone increasing sequence of $R$-covering sets and define

$$
\rho(f, g)=\sum_{n=1}^{\infty}\left(\frac{1}{2^{n}}\right) \frac{d_{n}(f, g)}{1+d_{n}(f, g)},
$$

where

$$
d_{n}(f, g)=\max _{z \in \bar{R}_{n}}|f(z)-g(z)| .
$$

Let $\Omega^{\prime}$ denote the topology induced in $R$ by the metric $\rho$ and designate the topological space thus defined by $\left(R, R^{\prime}\right)$. Now, a sequence $\left\{f_{n}\right\}$ in $\left(\Omega, \Omega^{\prime}\right)$ converges if and only if $\left\{f_{n}(z)\right\}$ converges uniformly on every compact set $K \subset R\left[3\right.$, p. 139]. The space $\left(R, R^{\prime}\right)$ is complete $[3$, p. 139]. It also has a property which is close to local compactness [3, p. 142], since any infinite uniformly bounded set of functions $\left\{f_{\alpha}(z)\right\}$ contains a sequence $\left\{f_{n}(z)\right\}$ which converges uniformly on any closed subset of $R$ and $\lim _{n \rightarrow \infty} f_{n}(z)$ is analytic in $R$. However, the

Presented to the Society, December 27, 1954; received by the editors January 27, 1955 and, in revised form, July 12, 1955 and April 25, 1956.

1 The author wishes to thank Professor P. W. Ketchum of the University of Illinois and Dr. C. Masaitis of the University of Tennessee for reading most of this paper.

2 Numbers in brackets refer to the bibliography at the end of the paper. 
sets whose compactness is established are not the closures of neighborhoods by the $R^{\prime}$-topology.

In this paper $\Omega^{\prime}$-pseudo local compactness is defined. The space $(R, R)$ is shown to be $R^{\prime}$-pseudo locally compact, where $R$ designates the set of functions analytic in an open region $R, R$ is the topology induced by the metric $d(f, g)=\sup _{z \in R}|f(z)-g(z)|$, and $\Omega^{\prime}$ is the topology defined in the preceding paragraph.

2. $R^{\prime}$-pseudo local compactness defined. Suppose $A$ is a point set for which two topologies $u$ and $\mathfrak{u}^{\prime}$ are defined. Thus, topological spaces $(A, \mathfrak{u})$ and $\left(A, \mathfrak{u}^{\prime}\right)$ are determined. If for every point $p \in A$ and an arbitrary $\mathcal{u}^{\prime}$-neighborhood $N_{p}\left(A, \mathcal{u}^{\prime}\right)$ of $p$ there exists an $\mathcal{U}$-neighborhood $N_{p}(A, \mathfrak{u}) \subset N_{p}\left(A, \mathfrak{u}^{\prime}\right)$, then $\mathcal{u}$ is said to be stronger than the $\mathfrak{u}^{\prime}$-topology $\left(\mathcal{u} \supset \mathcal{u}^{\prime}\right)$ or $\mathfrak{u}^{\prime}$ weaker than the $\mathcal{u}$-topology $\left(\mathcal{u}^{\prime} \subset \mathcal{u}\right)$ $[2$, p. 16]. We note that if the $\mathcal{U}$-topology is stronger a limit point by the $\mathcal{U}$-topology is necessarily a limit point by the $\mathfrak{u}^{\prime}$-topology.

In this paper, a topological space $T$ is understood to be compact if and only if every infinite subset of $T$ has at least one limit point in $T$. The following well known theorem is stated for reference.

Theorem A. If a point set $A$ is compact by a topology $u$, it is compact by any weaker $u^{\prime}$-topology $[2$, p. 16].

The closure in the space $(A, \mathcal{u})$ by the $u$-topology of a point set $E \subset A$ will be denoted by $\bar{E}(A, \mathcal{u})$, or in cases where the topology and space are evident simply by $\bar{E}$.

A topological space $(R, R)$ will be said to be $R^{\prime}$-pseudo locally compact, or pseudo locally compact by the $\mathbb{R}^{\prime}$-topology, if any neighborhood by the $R$-topology of an arbitrary point $p$ of $R$ contains some neighborhood $N_{p}(R, R)$ by the $R$-topology, such that the point set $\bar{N}_{p}(R, R)$ is compact by the $R^{\prime}$-topology.

We note that if a space $(R, R)$ is locally compact then $(R, R)$ is $R$-pseudo locally compact and hence $R^{\prime}$-pseudo locally compact by any weaker $\mathbb{R}^{\prime}$-topology. (See Theorem A.) However, a space $(R, R)$ may be $R^{\prime}$-pseudo locally compact but not locally compact by a stronger $R$-topology. (See paragraph 4 and the example in the next paragraph.) If there are two metric topologies $\Omega^{\prime}$ and $\Omega^{\prime \prime}$ by which $(R, \Omega)$ is pseudo locally compact and if $R^{\prime \prime} \supset R^{\prime}$, then $R^{\prime}$ and $R^{\prime \prime}$ are locally equivalent with respect to $(R, R)$. (See Corollary, Theorem 2.)

We consider an example of an $R^{\prime}$-pseudo locally compact space. ${ }^{3}$ Let $R$ be the complex plane. For the $R$-topology define a neighbor-

${ }^{3}$ This example was suggested by Professor R. H. Bing of the University of Wisconsin. 
hood of a point $p$ as a circular disc with $p$ as center except that all points, other than $p$ itself, having rational coordinates are omitted. Let $\Omega^{\prime}$ be the topology in which a neighborhood of $p$ is a circular disc with $p$ as center. The space $(R, R)$ is $R^{\prime}$-pseudo locally compact, but is not locally compact.

3. General theorems on $R^{\prime}$-pseudo locally compact spaces. In the following theorems it is to be understood that the $R$ or $R^{\prime}$-topology for a subset of $R$ is the relative topology.

We obtain a sufficient condition that a subset of an $R^{\prime}$-pseudo locally compact space be $R^{\prime}$-pseudo locally compact.

THEOREM 1. Let $R$ be a point set with a topology $R$ assigned, also a metric topology $\mathbb{R}^{\prime} \subset R \cdot{ }^{4}$ Suppose that $(R, R)$ is $R^{\prime}$-pseudo locally compact. Let $R^{*}$ be any subset of $R$. Then $\left(R^{*}, R\right)$ is $\Omega^{\prime}$-pseudo locally compact if $\left(R^{*}, R^{\prime}\right)$ is complete by the $R^{\prime}$-topology.

Proof. If $N_{q}\left(R^{*}, R\right)$ is any neighborhood of an arbitrary point $q$ of $R^{*}, N_{q}\left(R^{*}, R\right)=N_{q}(R, \Omega) \cap R^{*}$. By the hypothesis that $(R, R)$ is $R^{\prime}$-pseudo locally compact, $N_{q}(R, R)$ contains a neighborhood $N_{q}^{\prime}(R, R)$ such that $\bar{N}_{q}^{\prime}(R, R)$ is compact by the $R^{\prime}$-topology. We note that $N_{q}^{\prime}(R, R) \cap R^{*}$ is a neighborhood $N_{q}^{\prime}\left(R^{*}, \Omega\right)$ of $q$ in $R^{*}$.

We show that $\bar{N}_{q}^{\prime}\left(R^{*}, \Omega\right)$ is compact by the $R^{\prime}$-topology. Let $\left\{p_{r}\right\}$ be any infinite subset of $\bar{N}_{q}^{\prime}\left(R^{*}, \Omega\right)$. Then $\left\{p_{r}\right\} \subset \bar{N}_{q}^{\prime}(R, R)$ and hence by the $\mathcal{R}^{\prime}$-topology has a limit point $p \in \bar{N}_{q}^{\prime}(R, R)$. There is a sequence $\left\{p_{r_{i}}\right\} \subset\left\{p_{r}\right\}$ which converges to $p$ by the $R^{\prime}$-topology. Since $\left(R^{*}, \Omega^{\prime}\right)$ is complete, $p \in R^{*}$. Thus, we obtain that $p \in \bar{N}_{q}^{\prime}(R, R) \cap R^{*}$, which is just $\bar{N}_{q}^{\prime}\left(R^{*}, R\right)$.

This completes the proof that an arbitrary neighborhood $N_{q}\left(R^{*}, \mathbb{R}\right)$ of $q$ contains a neighborhood $N_{q}^{\prime}\left(R^{*}, \Omega\right)$ such that $\bar{N}_{q}^{\prime}\left(R^{*}, \Omega\right)$ is compact by the $R^{\prime}$-topology, that is, that $\left(R^{*}, R\right)$ is $R^{\prime}$-pseudo locally compact.

The following theorem is used in the proof of Theorem 2.

TheOREm B. For topologies $\boldsymbol{R}_{\mu} \subset R_{\nu}$ on a space $R$, if $R$ is compact by $\mathfrak{R}_{\nu}$ and a $T_{2}$-space by $R_{\mu}$, then $\mathfrak{R}_{\mu}$ is equivalent to $\mathfrak{R}_{\nu}$ (if $\mathfrak{R}_{\nu}$ is metric) ${ }^{5}$ [2, p. 28].

Let $R, R^{\prime}$, and $R^{\prime \prime}$ be topologies defined for a point set $R$. Then $\Omega^{\prime}$ and $\Omega^{\prime \prime}$ are said to be locally equivalent with respect to $(R, R)$ provided the following condition is satisfied: Every neighborhood by

4 Because of differences in usage of the terms weaker and stronger topologies the reader is reminded of the definitions in $\mathbb{2} 2$.

5 With the additional requirement that the topology is metric our definition of compactness is equivalent to that used by Nakano. 
the $R$-topology of an arbitrary point $p$ contains a neighborhood $N_{p}(R, \Omega)$ such that the relative $\Omega^{\prime}$, and $\Omega^{\prime \prime}$-topologies for the set $S=\bar{N}_{p}(R, R)$ (where the closure is by the $R$-topology) are equivalent.

Theorem 2. Suppose $(R, R)$ is $\mathbb{R}^{\prime}$-pseudo locally compact. Any metric topology $R^{\prime \prime} \supset R^{\prime}$ such that $(R, R)$ is $R^{\prime \prime}$-pseudo locally compact is locally equivalent to $R^{\prime}$ if $R^{\prime}$ is $T_{2}$; any $T_{2}$-topology $\Omega^{\prime \prime} \subset \Omega^{\prime}$ is locally equivalent to $\boldsymbol{R}^{\prime}$ if $\boldsymbol{R}^{\prime}$ is metric.

Proof. We first consider the case $R^{\prime \prime} \supset R^{\prime}$. Since $(R, R)$ is $R^{\prime \prime}$ pseudo locally compact, any neighborhood by the $R$-topology of a point $p$ contains a neighborhood $N_{p}(R, \Omega)$ such that the point set $S=\bar{N}_{p}(R, R)$ is compact by the $R^{\prime \prime}$-topology. That is, the space $\left(S, \mathbb{R}^{\prime \prime}\right)$ is compact. The hypothesis that $\left(R, \Omega^{\prime}\right)$ is $T_{2}$ implies that the subspace $\left(S, R^{\prime}\right)$ is $T_{2}$ by the relative $\mathbb{R}^{\prime}$-topology. Now Theorem $\mathrm{B}$ implies that the relative $R^{\prime}$ and $\Omega^{\prime \prime}$ topologies for $S$ are equivalent. We have shown that any neighborhood of an arbitrary point $p$ contains a neighborhood $N_{p}(R, \Omega)$ such that the relative $\Omega^{\prime}$ and $\Omega^{\prime \prime}$ topologies for the set $S=\bar{N}_{p}(R, R)$ are equivalent. That is, $R^{\prime}$ and $R^{\prime \prime}$ are locally equivalent with respect to $(R, R)$.

Next we consider the case $R^{\prime \prime} \subset \Omega^{\prime}$, where $\Omega^{\prime \prime}$ is $T_{2}$. Any neighborhood of an arbitrary point $p$ contains a neighborhood $N_{p}(R, R)$ such that $S=\bar{N}_{p}(R, R)$ is compact by the metric $R^{\prime}$-topology. The hypothesis that $\left(R, \mathbb{R}^{\prime \prime}\right)$ is $T_{2}$ implies that the subspace $\left(S, R^{\prime \prime}\right)$ is $T_{2}$. Now Theorem B implies that the relative $R^{\prime}$ and $\mathcal{R}^{\prime \prime}$ topologies for $S$ are equivalent. This completes the proof that any neighborhood contains a neighborhood $N_{p}(R, \Omega)$ such that the relative $\Omega^{\prime}$ and $\Omega^{\prime \prime}$ topologies for the set $S=\bar{N}_{p}(R, R)$ are equivalent, that is, that $\mathcal{R}^{\prime}$ and $R^{\prime \prime}$ are locally equivalent with respect to $(R, R)$.

COROLlARY. If there are two metric topologies $R^{\prime}$ and $\mathbb{R}^{\prime \prime}$, one stronger than the other, by which $(R, R)$ is pseudo-locally compact, then $\mathcal{R}^{\prime}$ and $R^{\prime \prime}$ are locally equivalent with respect to $(R, R)$.

4. $R^{\prime}$-pseudo locally compact spaces in analytic function theory. Let $R$ be an open region of the complex plane. Then a sequence of sets $\left\{R_{j}\right\}$ which satisfy the following conditions will be called a monotone increasing sequence of $R$-covering sets: (1) $R_{n} \subset R$; (2) $\bar{R}_{n}$ is interior to $R_{n+1} ;(3) \bigcup_{j=1}^{\infty} R_{j}=R$.

Suppose that $R$ is an open region of the complex plane and that $\left\{R_{j}\right\}$ is a particular monotone increasing sequence of $R$-covering sets. Let $S$ denote a set of functions which are meromorphic in $R$.

For each pair of functions $f$ and $g$ of $s$ let $I_{f o}$ denote the subset of $R$ on each point of which $f(z)$ or $g(z)$ has a pole. Then define a dis- 
tance function $d(f, g)$ as $\sup _{z \in\left(R-I_{f o}\right)}|f(z)-g(z)|$ for those $f, g \in S$ for which this is finite. Now let $R$ denote the topology so induced in $S$. We note that if $d(f, g)$ is defined, the poles of $f(z)$ and $g(z)$ in $R$ necessarily coincide and have the same principal parts. Hence, any neighborhood, also any closed neighborhood, of $f$ by the $R$-topology contains only. functions meromorphic in $R$ which have poles in $R$ identical with those of $f(z)$. Thus, if $g \in N_{f}(\mathcal{S}, R), f(z)-g(z)$ is analytic in $R$.

A different topology $\Omega^{\prime}$ is induced by the metric function

$$
\left.\rho(f, g)=\sum_{n=1}^{\infty}\left(\frac{1}{2^{n}}\right) \frac{d_{n}(f, g)}{1+d_{n}(f, g)},\right)
$$

where $d_{n}(f, g)=\sup _{z \in\left(R_{n}-I_{f g}\right)}|f(z)-g(z)|[3$, p. 139]. Now

$$
\sum_{n=1}^{\infty}\left(\frac{1}{2^{n}}\right) \frac{d_{n}(f, g)}{1+d_{n}(f, g)} \leqq \sum_{n=1}^{\infty}\left(\frac{1}{2^{n}}\right) \frac{d(f, g),}{1+d_{n}(f, g)} \leqq d(f, g) ;
$$

that is, $\rho(f, g) \leqq d(f, g)$. (If $d_{n}(f, g)$ is undefined, $d_{n}(f, g) /\left(1+d_{n}(f, g)\right.$ ) is to be taken as " 1 " in

$$
\left.\sum_{n=1}^{\infty}\left(\frac{1}{2^{n}}\right) \frac{d_{n}(f, g)}{1+d_{n}(f, g)} \cdot\right)
$$

Lemma. The R-topology is stronger than the $\mathbb{R}^{\prime}$-topology.

Proof. Corresponding to any point $f$ of $S$ and to any $\epsilon>0$, there is defined an $\epsilon$-neighborhood $N_{f}^{(\epsilon)}\left(S, \mathcal{R}^{\prime}\right)$ by the $\mathcal{R}^{\prime}$-topology and also an $\epsilon$-neighborhood $N_{f}^{(\epsilon)}(\mathcal{S}, \mathcal{R})$ by the $R$-topology. We show that $N_{f}^{(\epsilon)}(\mathcal{S}, \mathcal{R})$ $\subset N_{f}^{(\epsilon)}\left(\mathcal{S}, \mathbb{R}^{\prime}\right)$. If $g \in N_{f}^{(\epsilon)}(\mathcal{S}, \mathbb{R}), d(f, g)<\epsilon$. Since $\rho(f, g) \leqq d(f, g)$, $\rho(f, g)<\epsilon$ and so $g \in N_{f}^{(e)}\left(\mathcal{S}, \mathbb{R}^{\prime}\right)$.

The following theorem is similar to one stated by Thron [3, p. 139] for analytic functions. We shall say that a sequence $\left\{f_{n}(z)\right\}$ of functions meromorphic on $\bar{R}_{k}$ converges uniformly to $f(z)$ on $\bar{R}_{k}$, if, corresponding to any $\epsilon>0$, there exists $N$ such that $n>N$ implies $\left|f(z)-f_{n}(z)\right|<\epsilon$ on $\left(\bar{R}_{k}-I_{f, f_{n}}\right)$.

THEOREM $C$. Let $S$ be any infinite set of functions which are meromorphic in an open region $R$. A sequence $\left\{f_{n}\right\}$ in $\left(\mathcal{S}, \Omega^{\prime}\right)$ converges to $f$ in $\left(\mathcal{S}, \boldsymbol{R}^{\prime}\right)$ if and only if $\left\{f_{n}(z)\right\}$ converges uniformly to $f(z) \in \mathcal{S}$ on every $\bar{R}_{k}$.

Proof. Suppose $\left\{f_{n}\right\}$ converges to $f$ in $\left(\mathcal{S}, \boldsymbol{R}^{\prime}\right)$, that is, that $\lim _{n \rightarrow \infty} \rho\left(f_{n}, f\right)$, or

$$
\lim _{n \rightarrow \infty} \sum_{j=1}^{\infty}\left(\frac{1}{2^{i}}\right) \frac{d_{j}\left(f_{n}, f\right)}{1+\left\lfloor d_{j}\left(f_{n}, f^{*}\right)\right.}=0,
$$


where $f \in S$. It is sufficient to show that this implies $\lim _{n \rightarrow \infty} d_{k}\left(f_{n}, f\right)=0$ for every $k$.

We first show that, for arbitrary $k$,

$$
\lim _{n \rightarrow \infty} \frac{d_{k}\left(f_{n}, f\right)}{1+d_{k}\left(f_{n}, f\right)}=0 .
$$

If

$$
\lim _{n \rightarrow \infty} \frac{d_{k}\left(f_{n}, f\right)}{1+d_{k}\left(f_{n}, f\right)} \neq 0
$$

then for some $\eta>0$ and every $N$ there is $r>N$ such that

$$
\frac{d_{k}\left(f_{r}, f\right)}{1+d_{k}\left(f_{r}, f\right)}>\eta
$$

Then

$$
\rho\left(f_{r}, f\right)=\sum_{j=1}^{\infty}\left(\frac{1}{2^{j}}\right) \frac{d_{j}\left(f_{r}, f\right)}{1+d_{j}\left(f_{r}, f\right)}>\frac{\eta}{2^{k}} .
$$

Thus, for any $N$, there exists $r>N$ such that $\rho\left(f_{r}, f\right)>\eta / 2^{k}$. This contradicts the hypothesis that $\lim _{n \rightarrow \infty} \rho\left(f_{n}, f\right)=0$. We conclude that, for arbitrary $k$,

$$
\lim _{n \rightarrow \infty} \frac{d_{k}\left(f_{n}, f\right)}{1+d_{k}\left(f_{n}, f\right)}=0,
$$

and hence that $\lim _{n \rightarrow \infty} d_{k}\left(f_{n}, f\right)=0$, that is, that $\left\{f_{n}(z)\right\}$ converges to $f(z)$ uniformly on each $\bar{R}_{k}$.

To prove the converse we note that, if $\left\{f_{n}(z)\right\}$ converges uniformly to $f(z)$ on every $\bar{R}_{k}$, then $\lim _{n \rightarrow \infty}\left(d_{k}\left(f_{n}, f\right) /\left(1+d_{k}\left(f_{n}, f\right)\right)\right)=0$. There exists $N(k)$ so that $n>N(k)$ implies that

$$
\frac{d_{k}\left(f_{n}, f\right)}{1+d_{k}\left(f_{n}, f\right)}<\frac{\epsilon}{2^{k}} .
$$

Then

$$
\begin{array}{rlrl}
\rho\left(f_{n}, f\right) & <\sum_{j=1}^{k}\left(\frac{1}{2^{i}}\right) \frac{\epsilon}{2^{k}}+\sum_{j=k+1}^{\infty} \frac{1}{2^{j}} & \\
& =\frac{2^{k}-1}{2^{k}}\left(\frac{\epsilon}{2^{k}}\right)+\frac{1}{2^{k}} \quad \text { for all } n>N(k) .
\end{array}
$$

Thus, corresponding to any $\eta>0, k$ can be chosen so that when 
$n>N(k), \rho\left(f_{n}, f\right)<\eta$. Hence, $\lim _{n \rightarrow \infty} \rho\left(f_{n}, f\right)=0$, or $\lim _{n \rightarrow \infty} f_{n}=f$ in $\left(S, R^{\prime}\right)$.

THEOREM 3. Let $R$ be the set of all functions meromorphic in an open region $R$. Then $(R, R)$ is $R^{\prime}$-pseudo locally compact.

Proof. Let $f$ be any point of $R$; that is, let $f(z)$ be any function which is meromorphic in $R$. An $\epsilon$-neighborhood of $f$ by the R-topology is defined by

Then

$$
N_{f}(R, R)=\{g(z) \mid(1) g(z) \in R \text { and }(2) d(f, g)<\epsilon\} .
$$

$$
\bar{N}_{f}(R, Q)=\{g(z) \mid(1) g(z) \in R \text { and }(2) d(f, g) \leqq \epsilon\} .
$$

It is sufficient to show that any infinite subset $\left\{g_{\alpha}\right\}$ in $\bar{N}_{f}(\Omega, \Omega)$ has a limit point by the $R^{\prime}$-topology. We note that each function $g_{\alpha}(z)$ necessarily has poles coinciding with those of $f(z)$ in $R$ with the same principal parts as those of $f(z)$. Hence, $f(z)-g_{\alpha}(z)$ is analytic everywhere in $R$. We define $h_{\alpha}(z)=f(z)-g_{\alpha}(z)$ when $z \in R$. Since $\left|h_{\alpha}(z)\right| \leqq \epsilon$ when $z \in R,\left\{h_{\alpha}(z)\right\}$ is uniformly bounded on $R$. We have shown that $\left\{h_{\alpha}(z)\right\}$ is a set of functions analytic and uniformly bounded on $R$. Therefore, Ascoli's Theorem [4, p. 169; 3, p. 140] implies the existence of a sequence $\left\{h_{\alpha_{n}}(z)\right\}$ which is a subset of $\left\{h_{\alpha}(z)\right\}$ and which converges at each point of $R$, uniformly on each $\bar{R}_{n}$. By a well known theorem [4, p. 98] $\lim _{n \rightarrow \infty} h_{\alpha_{n}}(z)=h(z)$ is analytic in $R$. Moreover, since $\left|h_{\alpha_{n}}(z)\right|=\left|f(z)-g_{\alpha_{n}}(z)\right| \leqq \epsilon,|h(z)| \leqq \epsilon$.

Define $g(z)=f(z)-h(z)$. We note that, in $R, h(z)=\lim _{n \rightarrow \infty} h_{\alpha_{n}}(z)$ $=\lim _{n \rightarrow \infty}\left(f(z)-g_{\alpha_{n}}(z)\right)=f(z)-\lim _{n \rightarrow \infty} g_{\alpha_{n}}(z)$, where the convergence holds everywhere in $R$ (except at the points of $I_{f g}$ ) and uniformly on every $\bar{R}_{n}$. That is, $h(z)=f(z)-\lim _{n \rightarrow \infty} g_{\alpha_{n}}(z)$. Then, since $h(z)$ $=f(z)-g(z)$, we conclude that $g(z)=\lim _{n \rightarrow \infty} g_{\alpha_{n}}(z)$; hence, Theorem C implies that $\left\{g_{\alpha_{n}}\right\}$ converges to $g$ in $R$ by the $R^{\prime}$-topology. As was noted in the preceding paragraph, $|h(z)| \leqq \epsilon$; that is, $|f(z)-g(z)| \leqq \epsilon$. Then, since $g(z)$ is meromorphic in $R$ and, therefore, belongs to $R$, $g \in \bar{N}_{f}(R, R)$.

Thus, if $f$ is any point of $R$ and $N_{f}(R, R)$ is any neighborhood of $f$ by the $R$-topology, $N_{f}(R, R)$ contains a neighborhood-namely, $N_{f}(R, R)$ itself-such that any infinite subset $\left\{g_{\alpha}\right\}$ in $\bar{N}_{f}(R, \Re)$ contains a sequence $\left\{g_{\alpha_{n}}\right\}$ which converges by the $R^{\prime}$-topology to a point $g \in \bar{N}_{f}(R, R)$. This completes the proof that $(R, R)$ is $R^{\prime}$-pseudo locally compact.

Corollary. Any metric topology $\mathbb{R}^{\prime \prime}$, either stronger or weaker than $\Omega^{\prime}$, by which $(R, R)$ is pseudo locally compact is locally equivalent to $R^{\prime}$. 
Proof. This follows directly from the corollary of Theorem 2.

In the following theorems, unless indicated otherwise, it is to be understood that $R$ is an open region, $R$ is the set of all functions meromorphic in $R$, and that topologies $R$ and $\mathcal{R}^{\prime}$ are defined as before.

TheOREm 4. Let $R^{*}$ be a set of functions meromorphic in $R$. Then $\left(R^{*}, \Omega\right)$ is $\boldsymbol{R}^{\prime}$-pseudo locally compact if $\left(R^{*}, \Omega^{\prime}\right)$ is complete.

Proof. We note that $R^{*} \subset R$. By Theorem $3(R, R)$ is $R^{\prime}$-pseudo locally compact and by hypothesis $\left(R^{*}, \Omega^{\prime}\right)$ is complete. Now Theorem 1 implies that $\left(R^{*}, R\right)$ is $R^{\prime}$-pseudo locally compact.

Theorem 5. The subspace $\left(R^{(A)}, R\right)$ of $(R, R)$ whose points are those functions analytic everywhere in $R$ is $R^{\prime}$-pseudo locally compact.

Proof. According to a theorem stated by Thron [3, p. 139], $\left(\Omega^{(A)}, R^{\prime}\right)$ is complete. The required result follows from Theorem 4.

We next investigate the connection between a normal family of meromorphic functions and a set of meromorphic functions $R$ such that $(R, R)$ is $R^{\prime}$-pseudo locally compact. Of course, a normal family $R$ of functions meromorphic in a region $R$ can be extended to a compact normal family by adding to $R$ all of its limit functions [1, p. 183]. In the next theorem we suppose that $R$ is an infinite set of functions meromorphic in a region $R$ and show that if $(R, R)$ is $R^{\prime}$-pseudo locally compact then certain subsets of $R$ form normal families of functions.

Theorem 6. If $(\mathcal{R}, \mathbb{R})$ is $\mathfrak{R}^{\prime}$-pseudo locally compact, then every neighborhood by the $R$-topology of an arbitrary point $q$ of $R$ contains a neighborhood $N_{q}(R, R)$ such that the set of functions $\bar{N}_{q}(R, R)$ is a normal family of functions.

Proof. Choose $N_{q}(R, R)$ so that $\bar{N}_{q}(R, R)$ is compact by the $\Omega^{\prime}$-topology. Then every sequence $\left\{f_{n}\right\} \subset \bar{N}_{q}(R, R)$ has (by the $\mathbb{R}^{\prime}$ topology) a limit point $f \in \bar{N}_{q}(R, R)$. There exists a subsequence $\left\{f_{n_{i}}\right\}$ which converges to $f$ by the $R^{\prime}$-topology; that is, $\left\{f_{n_{i}}(z)\right\}$ converges to $f(z)$ everywhere in $R$ and uniformly on every closed subset of $R$.

\section{BiBLIOGRAPHY}

1. C. Carathéodory, Theory of functions of a complex variable, vol. 1, Chelsea Publishing Co., 1954.

2. H. Nakano, Topology and linear topological spaces, Maruzen Co., Ltd., 1951.

3. W. J. Thron, The theory of functions of a complex variable, Wiley, 1953. 1939.

4. E. C. Titchmarsh, The theory of functions, 2d ed., Oxford University Press, 